(2) Open Access Full Text Article

\title{
International Rotational Program of Emergency Medicine Residents to Mozambique: Introducing a Medical Education Program to a Single Hospital
}

This article was published in the following Dove Press journal: Open Access Emergency Medicine

\author{
Minsuk Sung' \\ Hoon Kim (D) ${ }^{2}$ \\ Dong Wun Shin $\mathbb{D D}^{2}$ \\ Woochan Jeon (iD) ${ }^{2}$ \\ Kyung Hwan Kim $\mathbb{D}^{2}$ \\ Hyunjong Kim (D) ${ }^{2}$ \\ Joon Min Park ${ }^{2}$ \\ Jung Eon Kim $\mathbb{D}^{2}$ \\ Junseok Park (D) ${ }^{2}$ \\ 'Department of Emergency Medicine, \\ Catholic Kwandong University \\ International St. Mary's Hospital, Incheon, \\ Korea; ${ }^{2}$ Department of Emergency \\ Medicine, Inje University Ilsan Paik \\ Hospital, Goyang, Korea
}

Correspondence: Junseok Park Juhwa-Ro 170, Goyang 10380, Korea Tel +82-31-910-7II9

Email jspark@paik.ac.kr
Introduction: There are several medical elective programs for low-income countries especially in medically vulnerable places. The Hospital Central de Quelimane (HCQ) is a regional hospital in Quelimane, capital city of the province of Zambezia in Mozambique. The HCQ serves as a regional base hospital for urgent and severe patients.

Methods: Four emergency medicine (EM) residents participated in our 2017-2018 rotational program for HCQ, to share medical knowledge with the local medical doctors and support the demands of medical equipment skills and educational programs. We determined the current capabilities of HCQ and designed a rotational program in accordance with the demands in the following areas: resuscitation, trauma, critical care, and radiology. We also introduced continuous education programs and administrative methods for future development of education.

Results: Throughout the four rotations of our EM residents, we conducted daily education and several practical lessons based on the demands of the local doctors and equipment operation. The educational program was administered by an educational administrator who was responsible for updating the medical and technical knowledge of doctors. With our programs, the doctors of HCQ were able to perform resuscitation and critical protocols, including manipulating equipment such as mechanical ventilator and defibrillator.

Conclusion: The rotation program by the four residents was successful, in terms of sharing medical knowledge and equipment management, and filling gaps identified in the operation of a modern hospital.

Keywords: international cooperation, Mozambique, developing countries, internship and residency

\section{Introduction}

Mozambique is a developing country in southern Africa. It holds a Human Development Index, the overall indicator of human development of a country, of 0.437, ranking $180^{\text {th }}$ among the countries in the United Nations (UN) in $2018 .^{1}$ The $\mathrm{UN}$ has reported a $76.8 \%$ poverty rate in Mozambique. Many health care and educational movements are progressing, but the country continues to see a high prevalence in maternal mortality, child mortality, and infectious diseases, such as HIV/AIDS, which lower life expectancy. When seen on a map, Mozambique is vertically stretched $2470 \mathrm{~km}$ along the southeast coast of Africa, consisted of mostly fertile agricultural lands, and most of the medical facilities are centered on the capital Maputo. ${ }^{2}$ As such, the opportunity for receiving medical services in 
remote areas is low, with economic and educational differences being other factors. Quelimane, the capital city of the province Zambezia, is about $1621 \mathrm{~km}$ away from Maputo; therefore, accessing medical service is a pressing issue. In the last decades, the Korean government has been reaching out to vulnerable areas in the African continent in which general medical services are not considered satisfactory compared with developed countries. Korean International Cooperation Agency known as KOICA offers grants to researchers and medical professionals to provide support to hospitals in medically vulnerable areas, such as HCQ., and as a participant, we started to support the Hospital Central de Quelimane (HCQ), which serves as a regional base hospital in Quelimane for emergent and severely ill patients. HCQ, which opened in October 2016, has 600 beds and a total of 664 employees to meet the demand of the medical requirements in this vulnerable province.

Meanwhile, there has been great interest in global emergency medicine (EM) in medically vulnerable lowincome countries (LICs) such as Mozambique. Several international rotation programs of medical doctors offer support in clinical training in LICs and also aim to build experience by participating in such programs. In a previous international rotation program, ${ }^{3}$ EM residents from the United States were sent to Argentina with structured goals and settings, and they practiced and focused on the EM setting in different pre-hospital systems, gaining experience that they would not be able to learn in the United States. Another program developed a global EM course in Rwanda ${ }^{4}$ to enable EM residents to observe the global settings of emergency care in LICs. They designed the emergency medicine in the tropics (EMIT) curriculum, which includes pre-hospital systems, EM, and prevention medicine. Through this course, the EM residents were challenged to share their knowledge and experience with the local specialists. Many surgical elective courses ${ }^{5-7}$ have also been developed through programs similar to the abovementioned. Other previous rotation programs have been based on the global programs that include the training course and research of EM residents. ${ }^{3,4}$ We focused on the support and enhancement of human resources at HCQ, particularly in terms of medical knowledge and management skills of medical equipment. Medical topics were selected based on the needs of the medical topics that the doctors at HCQ wanted to learn. As many global programs are based on opportunities for volunteerism, which lacks defined structured goals and systems, the present study aimed to show the effectiveness of a global rotation with set goals.

HCQ has been provided with new equipment and technical knowhow from other nations. Most of its human resources are from local clinics, as well as the province and neighboring countries. At a glance, the operability of HCQ was limited owing to limitations in human resources, such as skills to operate new equipment (e.g., ultrasonography, electrocardiogram) with applicable medical knowledge. Thus, we designed an education program based on bedside practice. In the designed program, every participating doctor could gain experience in using medical equipment they previously had no opportunity to operate. Therefore, to accomplish our goal, we had to design a new educational program that could be maintained by HCQ personnel by themselves so that the future doctors of HCQ can be trained by their own local educational program. Our program was set up as an initial roadmap; we designed plans in which four of our senior EM residents in Korea would serve in HCQ to support in human resource improvement and provide training in global EM. Ultimately, a welldesigned educational program would make local specialists capable of conducting succeeding education and developing programs for themselves based on future feedback from their doctors. Thus, continuity would be ensured. This program was expected to contribute to the improvement of the overall medical quality in Mozambique.

\section{Methods}

Our program was from July 14, 2017 to August 31, 2018. The rotation program of four EM residents was conducted as a specialist deployment (Table 1). We initially focused on the medical education programs of HCQ and suggestions for long-term continuity, and we also considered the environmental conditions that can affect the quality and objectives of the education program itself. Our educational program was designed for the training of medical equipment operation and introduction of basic medical principles applicable to facilities such as the intensive care unit (ICU) and emergency room (ER), both departments being considered crucial, especially given the position of HCQ in Mozambique.

Before starting the program, we worked on the current physical capabilities of HCQ and assessed its human resources. The assessments were made through investigating the medical personnel and environment based on 
Table I Four EM Residents Attendance and Goals

\begin{tabular}{|c|c|c|c|}
\hline Resident & Period & Common Goals & Personal Goals \\
\hline MG Kong & July 31 -August 18,2017 & Resuscitation Airway management & CPR training and setup hospital capabilities \\
\hline KC Lee & August 28-September 15, 2017 & Personnel education & Emergency cart setup ER and ICU settings \\
\hline SH Kim & April 23-May II, 2018 & & $\begin{array}{l}\text { Intubation education in cooperation with } \\
\text { anesthetist, Practical skills }\end{array}$ \\
\hline MS Sung & May 21 -June 08,2018 & & $\begin{array}{l}\text { Medical education and practice, Future } \\
\text { education plans }\end{array}$ \\
\hline
\end{tabular}

Abbreviation: CPR, cardiac pulmonary resuscitation.

Table 2 The Demands on the Emergency and Critical Care Capabilities of ER and ICU of HCQ

\begin{tabular}{|l|l|}
\hline Physical Capabilities & Human Capabilities \\
\hline Drug supplements & Electrocardiogram reading \\
Advanced airways & Emergency radiologic \\
& interpretation \\
Mechanical ventilators & Bedside ultrasonography \\
Laboratory results & Rapid sequence intubation \\
X-rays and computed & Ventilator management \\
tomography & \\
Ultrasonography & Cardiopulmonary resuscitation \\
\hline
\end{tabular}

current conditions of the wards, ICU, and ER. Details of the program are given in Table 2.

We planned on conducting a daily group lecture in the mornings for the education of doctors, and practical lessons, including bedside teaching and practices (e.g., equipment operations) in the afternoons. Each day, all available doctors were required to participate in the lessons, and their attendance was checked by the administrative director of education, recorded in an attendance sheet containing the date and doctors' name, department, and signature. The lessons and practice were about 1-hr long each, and a feedback survey was done after each. The feedback was accumulated for future education development. Table 3 lists the details of the lectures.

\section{Results}

The previous capabilities of HCQ were evaluated in several ways. Overall, plenty of patients admitted to the ER without any standardized charts and history protocols, and the patients were not efficiently differentiated based on triage basics.

Diagnostic equipment, such as ultrasonography and ECG, was available at the bedside, and infusion pumps and mechanical ventilators were ready for use. However, they were not fully utilized because of insufficient managing knowledge and experience of each medical doctor. A few emergency carts, also called crash cart or code cart (a set of trays on wheels used for transportation and dispensing of emergency medication and equipment at the site of emergent situation, such as CPR), were not made up for immediate situations, such as cardiac arrests and respiratory failures.

The vital signs of hemodynamically unstable patients were not being monitored periodically. Checking of vital signs was performed occasionally by only one pulse oximetry monitor without any ECG monitors attached; thus, the critical indicators, such as the heart rate, were only able to be assessed by indirect measures from the pulse oximetry. The monitoring devices were able to display arterial blood pressure and central venous pressure; however, these monitoring methods were not performed owing

Table 3 Lecture Topics of Regular Educational Conferences

\begin{tabular}{|l|l|l|l|}
\hline Resuscitation & Medical Knowledge & Radiography & Skills \\
\hline Basic Life Support & Hypertension & Chest X-ray & Electrocardiogram \\
ACLS & Diabetes Mellitus & Chest US & E-FAST \\
Basic and Advanced Airway & DKA & Chest CT & Intubation \\
Rapid Sequence Intubation & Arrythmia & Brain CT & Practical medical math \\
Mechanical Ventilators & Myocardial Infarction & Abdominal X-ray & \\
& Strokes & Abdominal US & \\
& Shock management & & \\
\hline
\end{tabular}

Abbreviations: ACLS, Advanced Cardiac Life Support; CT, Computed Tomography; DKA, Diabetic Ketoacidosis; E-FAST, Extended Focused Assessment with Sonography in Trauma; US, Ultrasonography. 
to lack of proper instruments and medical skills. The medical records of patients were written in mixed languages, such as Portuguese and Spanish, according to the doctor's preference; thus, they required a longer time to be read. A picture archiving and communication system was not available in the ER or ICU, so the doctors could not view patients' radiological images. A portable X-ray device was available, but the portable images were never taken because there was no protocol for portable X-rays. The arterial blood gas analysis (ABGA) machine was available without sampling kits, so the ABGA laboratory results were also unavailable. Thus, mechanical ventilation and post-intubation in critical patients were systemically limited in their supposed use. For certain situations, such as cardiac arrest and respiratory failure, no systemic protocols existed in HCQ. The doctors lacked the medical knowledge and experience to perform basic life support (BLS) and advanced cardiac life support (ACLS). They were also not aware of the importance of these protocols in hospital.

Each department in HCQ managed its own resources, such as medical equipment and drugs, without central control, resulting in non-even distribution of medical resources. This setup could be a problem in an emergency case, especially when a patient urgently needs a drug that is not currently available in the ER but available in the operating room.

There were many cases where a clinical physician would request a radiologist for image reading, but the information given to the radiologist was too limited, rendering the reading unhelpful. For example, the patient ID, name, and type of radiologic imaging would be given to the radiologist, who would give too-short readings that lack clinical correlation with the radiologic images. This practice will eventually block necessary communication channels between clinical doctors and radiologists. The abovementioned case is only one example of communication issues between distinct departments. There were also many similar problems within a department. For example, there was no regular conference or discussion that was programmed and managed. Doctors and nurses tended not to discuss patients or shared their knowledge.

At the time of the study, HCQ had a total of 37 specialist doctors, 25 of whom were from Cuba. Cuba has remarkable experience in international medical collaboration; they have more than 50,000 health professionals worldwide, working in conjunction with local health care professionals and significantly contributing to the improvement of deprived areas. ${ }^{8}$ The Cuban doctors in HCQ were all specialists and worked in various departments. They were taking care of the critical areas and rendering support to local general physicians, in clinical ways. However, the communication between the Cuban and local doctors was not frequent; indeed, discussions and educational meetings were rare.

Our 2017-2018 rotation program of EM residents included four doctors enrolled in the EM department of Ilsan Paik Hospital of Inje University. We mainly worked on current medical issues in HCQ, such as CPR, advanced airway management, and education and discussion sessions. We designed several repetitive procedural programs in which medical personnel in HCQ could participate in BLS and ACLS training. We collected the in-training tests and feedback of each participant so that we could evaluate procedural trial and errors, and subsequently formulate an improved educational program.

The importance of BLS and ACLS was acknowledged by the health workers in HCQ. However, owing to the lack of CPR experience with actual patients, the overall procedure of HCQ personnel was not satisfactory at the initial stage. Introducing BLS and making the participants capable of basic resuscitation were set as one of our goals. We performed several CPR practice sessions using mannequin models, such as Resusci Anne, in our lessons, as shown in Figure 1. We grouped several teams composed of small numbers and simulated in-hospital cardiac arrest situations. The participants were enthusiastic about the program and showed improvement and proficiency after each session. It was not uncommon to find actual CPR cases in HCQ, and the HCQ health workers working in departments, such as

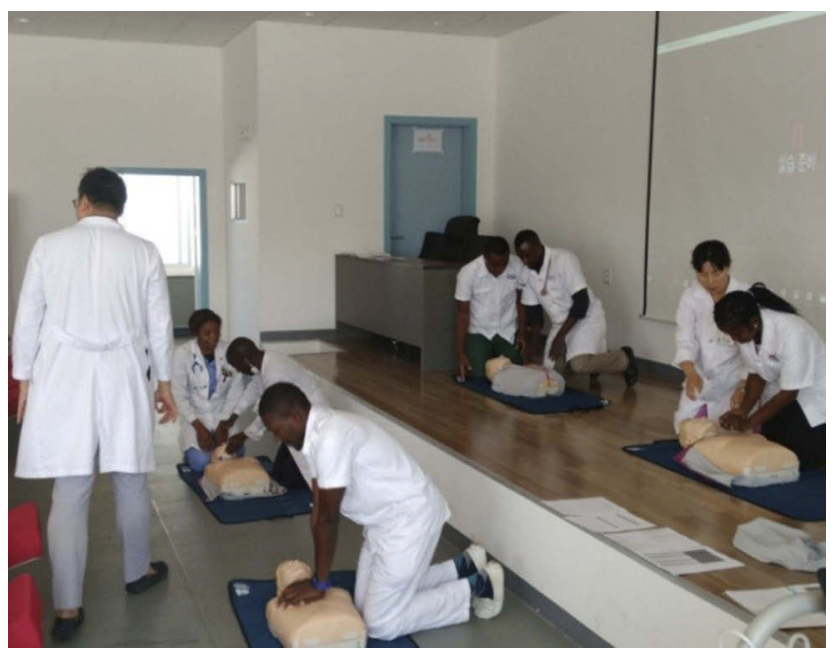

Figure I Group-based education in basic life support. 
ER and ICU, tried to provide CPR in cases where they did not provide effective resuscitation in the past.

Procedural skills, such as advanced airway management, are also considered extremely important in critical departments, such as the ER and ICU, as the acute survival of a patient could be greatly influenced by a successfully secured airway. HCQ had a few mechanical ventilators, and only the specialists in the anesthesia department were capable of practicing patient intubation. Doctors in the ER and ICU did have a general idea of intubation and mechanical ventilations. However, they were not able to apply their knowledge into practice. Simply teaching textbook concepts of advanced airway management was not enough to solve this problem. Our goal was geared toward the long term, in which the doctors should not only enhance their skills but also be able to teach other local doctors through continuous education. At the beginning, we focused on the procedural aspect of airway management, which, in most cases, is intubation. We introduced the intubation practice model as shown in Figure 2: the participants were able to check the anatomical structures in the airway and grasp the direct feedback result of their intubation procedure by visually inflating the artificial lungs. The doctors also had several chances for intubating actual patients in Figure 3, and this procedural education was considered effective at the first stage.

In actual patients, the doctors needed to consider rapid sequence intubation (RSI) and manage the mechanical ventilator. The majority of general physicians working in the ER and ICU had almost no experience in ventilator manipulation, although some of them had basic knowledge from textbooks or by indirect experience.

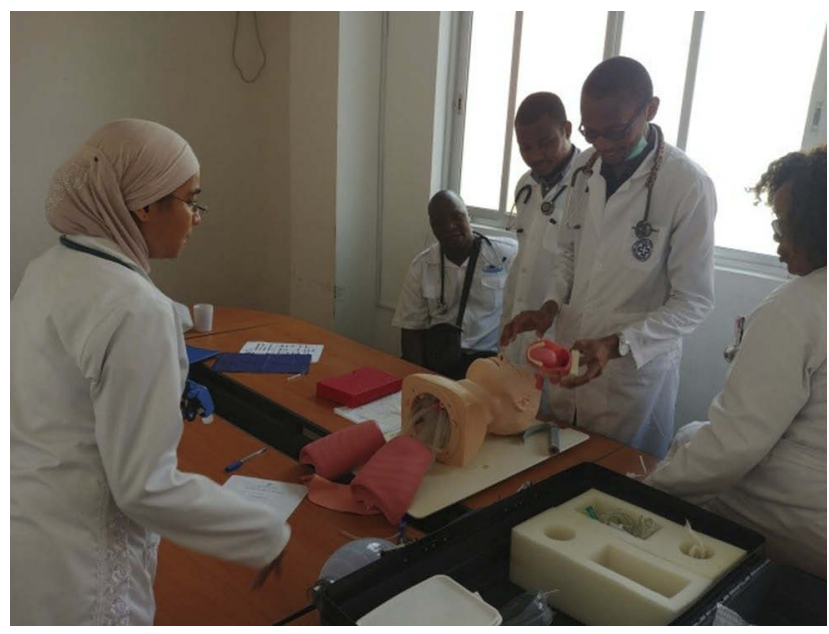

Figure 2 An intubation training model practice.

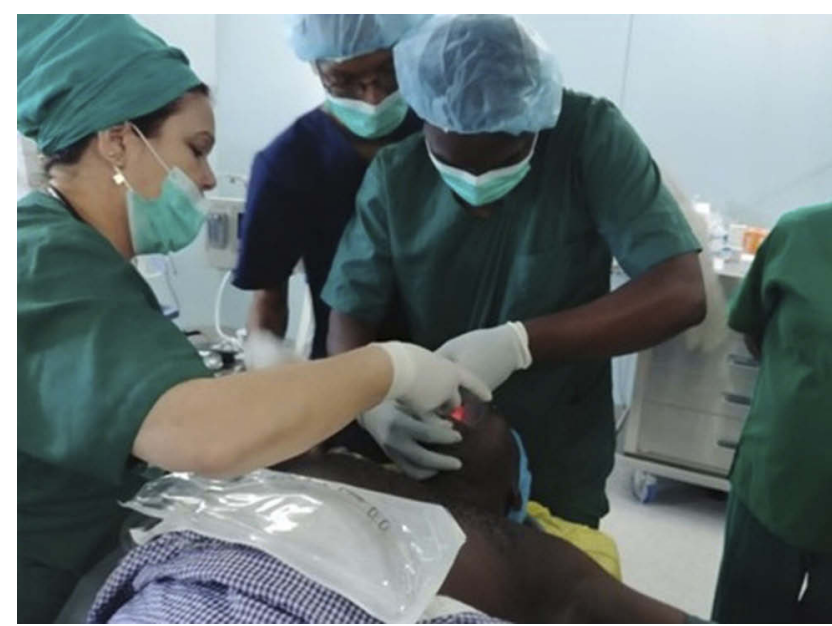

Figure 3 Endotracheal intubation training session in the operating theatre.

For the second stage of our program, we focused on the stage after successful intubation. For a successful RSI, the drugs used should be available, and the doctors must be aware of the correct usage. ${ }^{9}$ However, HCQ had limited drugs for induction and paralysis. For example, agents like etomidate and succinylcholine, which are considered safe and widely used in RSI, were not available. We had to find alternative drugs, and selected ketamine and rocuronium instead. The RSI protocol itself was not a complicated process, but the team work between the doctors and nurses played a significant role in the success of the entire procedure. Nurses working in the ER and ICU were training in using the infusion pump, as the infusion speed of drugs had to be precisely controlled by an infusion pump via an intravenous line. The doctors also needed to know how to convert the conventional units (ug/ $\mathrm{kg} / \mathrm{min})$ into the machine-dependent settings (cc/hr). These education sessions were done concomitantly. Continuous infusion of sedative agents for patients who were under mandatory ventilator modes was successfully set up and being administered by HCQ personnel, as a result of our ventilator lectures and practice. Moreover, the utilization of mechanical ventilators became more frequent, especially for severely ill patients in the ER and ICU.

In the activation of BLS and ACLS, including advanced airway management and mechanical ventilation utilization, critical drugs and equipment needed to be stored properly for easy and fast access. The emergency cart was not properly set up, and the placement of essential drugs was incorrect. There were also cases of the drugs being expired. The proper setup for the emergency cart was a definite preceding condition for all cases. We introduced the setting protocol of 


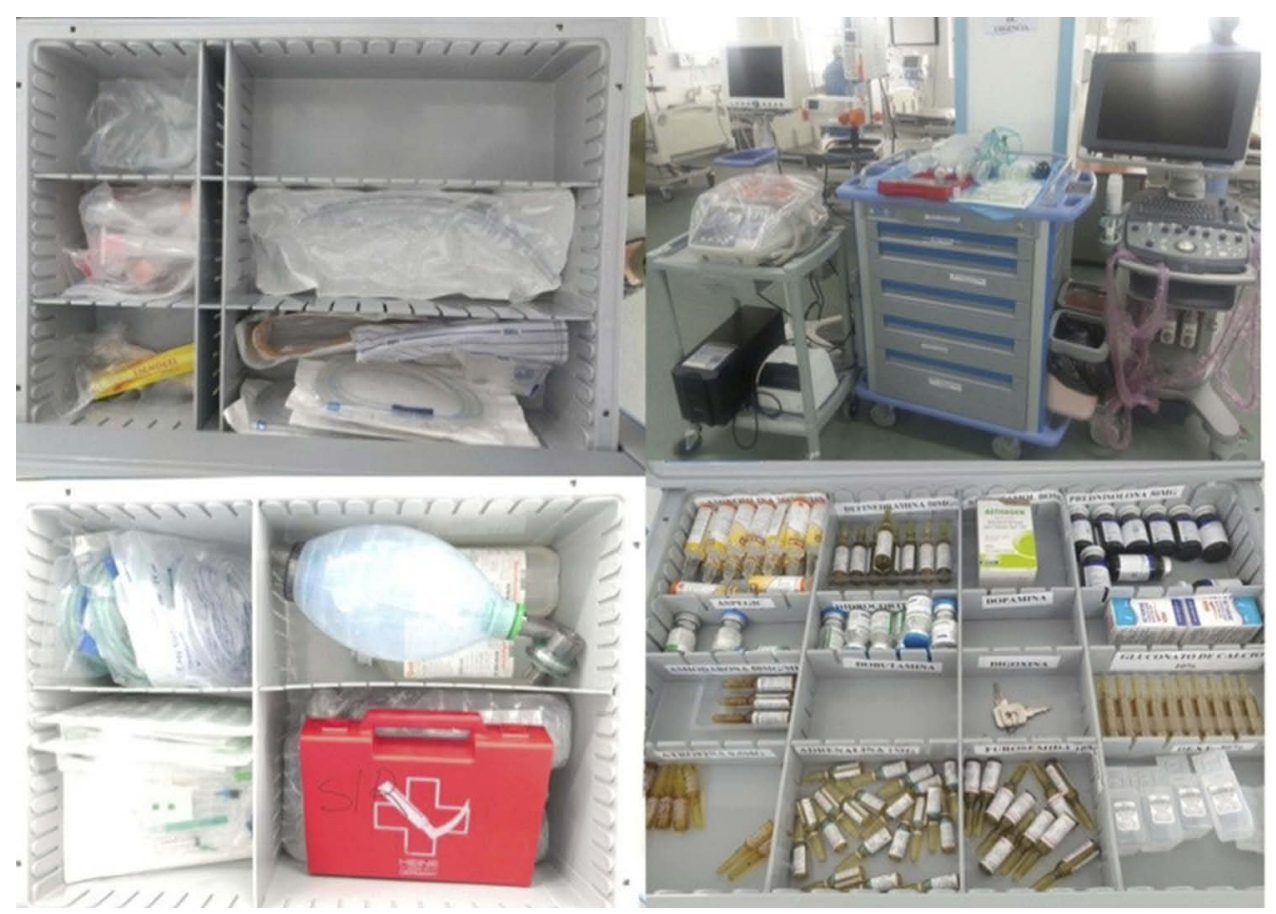

Figure 4 Emergency cart.

the emergency cart, placed in their necessary positions in the ER and ICU, as shown in Figure 4.

There were also many working specialists in HCQ from other countries, but most of the doctors were from Mozambique. There was no training course for EM residents, and most of the patients were cared primarily by local general practitioners. The educational programs for the medical personnel of HCQ were limited by the short history of the hospital and the lack of long-term technical support. The long-term continuity of education could only be achieved by a persistent program that should be designed in a systemic manner and based on feedback. HCQ was in need of an education and training department, with an administrative director responsible for development and management.

Conference meetings were held on Mondays and Fridays, and doctors' meetings were held on Wednesdays. These regular meetings were mostly on topics of current events and administrative reports. In view of medical support and skills improvement of the local doctors, we had to establish a new environment for medical educational purposes. We selected various topics in medical lectures held mandatorily every morning at 07:30, because we needed to make the participants become accustomed to the new education system and encourage them to participate in the lectures. The attendance rate was not satisfactory in the first few lectures. Less than half of the personnel showed up in the lecture, according to the recorded attendance sheet. Participants indicated in the feedback after the lesson that they were simply not interested in the medical topics, which did not seem to be immediately applicable in the field. Another feedback was from doctors, who mainly thought that the lectures were useless because the hospital lacked the medical resources to apply the lessons from the lecture and that the lectures wasted their time.

Simply putting all the lectures in a traditional didactic manner did not seem to work. Thus, we designed a separate education program, called the practice meetings, which were held by small groups of available doctors at a time. Each program was held in places such as the $\mathrm{CT}$ room, X-ray room, ER, and ICU. Each program consisted of two features: a basic slide lecture in the morning and a practice lecture in the afternoon. The practice lecture was designed in small groups so that everyone could handle the equipment. As shown in Figure 5, every participating doctor performed the focused assessment with sonography in trauma with one another. They shared their experiences and gave comments. This method was considered effective because the participants showed great enthusiasm; they introduced this process to other doctors, as well as prepared feedback and future demands.

All of the participants were required to sign in and out to check their attendance. The attendance sheet showed an 


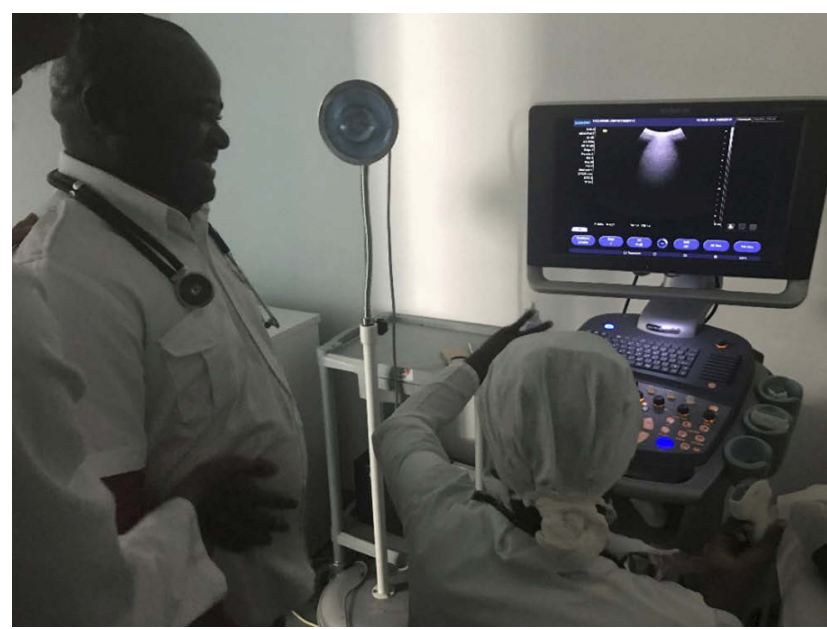

Figure 5 FAST examination.

increasing number of the concerns of the HCQ doctors, and they started to take the education program as a part of their day. The equipment and medical resources were reevaluated after every education session, and the report and feedback were then passed to the administrative director. This program was continuously done every week.

\section{Discussion}

Our program was an elective international rotational program, in which four of our enrolled EM residents were introduced to the medical educational system in and shared a learning experience with local doctors of HCQ. Many international programs supporting medical assistance to places of vulnerability, such as areas in Mozambique, have been implemented. Indeed, interest in international rotational programs has been increasing, and a resident survey conducted by an EM department in Korea ${ }^{10}$ showed that Korean EM residents have shown great interest in them. However, the current education and formal training of Korean EM residents offer limited opportunities for international rotational programs. In our program, four EM residents experienced how to set up and support the emergency medical skills, such as BLS, ACLS, and mechanical ventilation, in LICs hospitals, and also shared medical knowledge with local doctors.

HCQ in Quelimane, Mozambique, is the largest medical facility in the province of Zambezia, and is also supported by the central government of Mozambique. The systems and education of the human resources were insufficient for the equipment, such as mechanical ventilators and infusion pumps, and potential capability of their facilities to be fully utilized, thereby lowering the overall quality of the medical services provided. Therefore, the education of medical service personnel is crucial. In this context, the opportunities of having experienced personnel from other countries with a developed medical environment share their knowledge to local medical doctors are thought to be invaluable. To improve the quality of medical services in medically vulnerable areas, such as Quelimane, the local doctors need to share information on local characteristics, such as vulnerable populations, extent of governmental support, and available medical services and personnel, because the medical experts from other countries may not be aware of the specific local environmental conditions. Despite the duration of each rotation being limited to three weeks, we performed and setup education programs and conducted lectures and equipment practice during our total of four rotations of EM residents. Continuity and development of such education cannot be ensured unless local doctors provide practical training themselves. Progressive educational programs should be started along with providing the lectures. Moreover, one-way lectures and practice will not suffice; therefore, we suggested that a local doctor be designated to carry the responsibilities of an education administrator. The education administrator will arrange future lectures and develop succeeding programs based on the data of the current education program, including feedback by the participants. The education administrator should be responsible for future educational programs.

\section{Limitations}

The current rotation program showed a few limitations. First, HCQ was not intended to be an educational hospital. The medical resources of the hospital were supported by the central government of Mozambique, in terms of equipment, but not human resources. The HCQ personnel consisted of professionals from various countries, and the majority of doctors were often replaced by other doctors from other facilities. Even in the situation where successful educational programs were made, a new set of doctors would be assigned present and the entire education process need to be re-conducted. Structured educational programs supported by the central government may be considered. Implementing this suggestion will help ensure that succeeding rotation programs and the education program will work together and consequently enhance the capabilities of HCQ. 
Second, the cultural and language differences between the local doctors and our EM residents proved to be a disadvantage to smooth communication. Our EM residents were not able to communicate in Portuguese, the primary language spoken in Mozambique. Thus, communication required two-way translation; for example, from Korean to English, and then to Portuguese, and vice versa. The medical terms were different between English and Portuguese, and we found that this led to misunderstandings.

Lastly, our rotation program was performed by each EM resident doctor. We set our programs based on the information from preceding experience; making adjustments for local situations was inevitable. This resulted in the need to make duplicated lecture topics and inefficient lecture schedules. Moreover, we had only the experience of four individual residents, which is not sufficient for formulating precise education plans. Future work should consider a thoroughly designed rotational program focused on education and management.

\section{Conclusion}

The rotation program by the four residents was successful, in terms of sharing medical knowledge and equipment management, and filling gaps identified in the operation of a modern hospital.

\section{Data Sharing Statement}

The data used to support this article are available from the corresponding author upon request.

\section{Funding}

This work was supported by a grant from Research year of Inje University in 2011 (20110135).

\section{Disclosure}

Professor Junseok Park report grants from INJE University, outside the submitted work. The authors declare that they have no other conflicts of interest regarding this paper.

\section{References}

1. United Nations Development Programme. Human development reports, human development indicators, Mozambique. Available at: http://www. hdr.undp.org/en/countries/profiles/MOZ. Accessed September 23rd, 2018.

2. UNDP around the world, Mozambique. Available at: http://www.mz. undp.org/content/mozambique/en/home/countryinfo.html. Accessed September 23rd, 2018.

3. Hayden SR, Valderrama CM, Xu M, Curran MA, Mazondo R, Soliman MA. Development of an international elective in an emergency medicine residency. $J$ Emerg Med. 2016;50(1):153-158. doi:10.1016/j.jemermed.2015.06.015

4. Yi S, Umuhire OF, Uwamahoro D, Guptill M, Cattermole GN. Developing and implementing a global emergency medicine course: lessons learned from Rwanda. Educ Health (Abingdon). 2017;30 (3):203-210. doi:10.4103/efh.EfH_72_17

5. Cintolo-Gonzalez JA, Bedada AG, Morris J, Azzie G. An international surgical rotation as a systems-based elective: the Botswana-University of Pennsylvania surgical experience. $J$ Surg Educ. 2016;73(2):355-359. doi:10.1016/j.jsurg.2015.09.019

6. Elobu AE, Kintu A, Galukande M, et al. Evaluating international global health collaborations: perspectives from surgery and anesthesia trainees in Uganda. Surgery. 2014;155(4):585-592. doi:10.1016/j.surg.2013.11.007

7. Jarman BT, Cogbill TH, Kitowski NJ. Development of an international elective in a general surgery residency. J Surg Educ. 2009;66 (4):222-224. doi:10.1016/j.jsurg.2009.07.003

8. Castello Gonzalez M, Pons Vasquez R, Rodriguez Bencomo D, Choonara I. International medical collaboration: lessons from Cuba. Children (Basel, Switzerland). 2016;3(4):20.

9. Okubo M, Gibo K, Hagiwara Y, Nakayama Y, Hasegawa K. The effectiveness of rapid sequence intubation (RSI) versus non-RSI in emergency department: an analysis of multicenter prospective observational study. Int J Emerg Med. 2017;10(1):1. doi:10.1186/s12245017-0129-8

10. Kong MG, Kim H, Park JS, et al. Awareness and attitude on international emergency medicine: a resident survey. Korean $J$ Emerg Med. 2018;29(2):127-35.
The Open Access Emergency Medicine is an international, peerreviewed, open access journal publishing original research, reports, editorials, reviews and commentaries on all aspects of emergency
medicine. The manuscript management system is completely online and includes a very quick and fair peer-review system, which is all easy to use. Visit http://www.dovepress.com/testimonials.php to read real quotes from published authors. 